\title{
On Chebyshev-Type Quadratures
}

\author{
By Walter Gautschi and Hiroki Yanagiwara
}

\begin{abstract}
According to a result of S. N. Bernstein, $n$-point Chebyshev quadrature formulas, with all nodes real, do not exist when $n=8$ or $n \geqq 10$. Modifications of such quadrature formulas have recently been suggested by R. E. Barnhill, J. E. Dennis, Jr. and G. M. Nielson, and by D. Kahaner. We establish here certain empirical observations made by these authors, notably the presence of multiple nodes. We also show how some of the quadrature rules proposed can be constructed by solving single algebraic equations, and we compute the respective nodes to 25 decimal digits. The same formulas also arise in recent work of P. Rabinowitz and N. Richter as limiting cases of optimal Chebyshev-type quadrature rules in a Hilbert space setting.
\end{abstract}

1. Introduction. The quadrature rule with equal coefficients,

$$
\int_{-1}^{1} f(t) d t=\frac{2}{n} \sum_{k=1}^{n} f\left(t_{k}\right)+R_{n}(f)
$$

is called a Chebyshev quadrature formula if it has polynomial degree $n$, i.e., if $R_{n}\left(t^{i}\right)=0$ for $i=0,1, \cdots, n$. (The requirement for $i=0$ is automatically satisfied.) By a classical result, due to $\mathrm{S}$. N. Bernstein [3], Chebyshev quadrature formulas with all nodes $t_{k}$ real do not exist if $n=8$ or $n \geqq 10$. Even if Chebyshev's requirement is relaxed to $R_{n}\left(t^{i}\right)=0, i=0,1, \cdots, p, p<n$, we can have $t_{k}$ all real in (1.1) only if (S. N. Bernstein [3])

$$
p<4 n^{1 / 2} .
$$

In the light of these negative results it is natural to consider the following two problems.

Problem I. Let $p$ and $q$ be integers such that $0 \leqq p<n \leqq q \leqq \infty$. Determine real nodes $\left\{t_{k}\right\}_{k=1}^{n}$ (hopefully contained in the interval $[-1,1]$ ) such that

$$
\sum_{j=p+1}^{q}\left[R_{n}\left(t^{i}\right)\right]^{2}=\min
$$

subject to

$$
R_{n}\left(t^{i}\right)=0, \quad j=1,2, \cdots, p .
$$

(The constraints (1.4) drop out if $p=0$.)

Problem I' ("symmetric problem"). Same as Problem I, but with the additional symmetry constraints

$$
t_{n+1-k}=-t_{k}, \quad k=1,2, \cdots, n .
$$

If $q=n$, and $n \leqq 7$ or $n=9$, Problems $\mathrm{I}$ and $\mathrm{I}^{\prime}$ are solved by the

Received January 11, 1973.

AMS (MOS) subject classifications (1970). Primary 65D30; Secondary 41A55.

Key words and phrases. Optimal quadrature formulas of Chebyshev type.

Copyright (c) 1974, American Mathematical Society 
classical Chebyshev formulas, which drive the objective function in (1.3) to zero. We assume therefore, in the following, that $n=8$ or $n \geqq 10$. Problems $\mathrm{I}$ and $\mathrm{I}^{\prime}$ for $p=0$ are considered by R. E. Barnhill et al. [2], Problem $\mathrm{I}^{\prime}$ for $q=n, p \neq 0$ by D. Kahaner [4]. Their approach is largely computational. Some of the results reported in [2] seem to indicate that Problem I in fact has a symmetric solution, but this remains unproved. All we show (Theorem 2.1) is that the solution to Problem $I$ is symmetric if it is unique. Another empirical observation made in [2], [4] is the presence of multiple nodes. We prove (Theorems 2.2 and 3.1) that Problems I and $I^{\prime}$, in the case $q=n$, indeed give rise to multiple nodes. We subsequently concentrate on Problem I' with $p=n-1$ (if $n$ is even), $p=n-2$ (if $n$ is odd), and show how its solution can be reduced to the solution of single algebraic equations (Theorem 3.2 and Section 4). Computational results, based on these procedures, reveal (Section 5) that the problem again cannot be solved if $n=12$ or $14 \leqq n \leqq 19$. By (1.2) it has no solution for $n \geqq 20$ either. For the remaining values of $n(n=8,10,11,13)$ the optimal nodes are computed to 25 decimals. They have previously been obtained (to 12 decimal digits) in a different context by P. Rabinowitz and N. Richter [5].

2. Problem I. In this and the subsequent sections we denote by $m_{j}$ the moments

$$
\begin{aligned}
m_{j}=\int_{-1}^{1} t^{i} d t & =0 & \text { if } j \text { is odd, } \\
& =2 /(j+1) & \text { if } j \text { is even. }
\end{aligned}
$$

THEOREM 2.1. If the solution to Problem I is unique (up to a permutation), then it is symmetric.

Proof. We assume the nodes ordered, say,

$$
t_{1} \geqq t_{2} \geqq \cdots \geqq t_{n}
$$

Our assumption then implies that Problem I has exactly one solution $\mathrm{t}=\left[t_{1}, t_{2}, \cdots, t_{n}\right]$ satisfying (2.1). Now, if $t$ is a solution of Problem $I$, then so is $-t$. Indeed, neither the objective function in (1.3), nor the constraints (1.4) are affected if $t$ is replaced by $-t$, the remainder $R_{n}\left(t^{j}\right)$ either remaining the same ( $j$ even), or merely changing sign ( $j$ odd). Consequently, if $(2.1)$ represents a solution, so does

$$
-t_{n} \geqq-t_{n-1} \geqq \cdots \geqq-t_{1} .
$$

By the assumed uniqueness, it follows that $t_{n+1-k}=-t_{k}, k=1,2, \cdots, n$, that is, symmetry as asserted.

THEOREM 2.2. Let $q=n$, and $n=8$ or $n \geqq 10$. If the constraints (1.4) admit real solutions, then every solution of Problem I has at least one multiple node.

Proof. We observe, first of all, that under the assumptions made, Problem I has a solution, and that any solution must occur at a finite point $\mathrm{t}=\left[t_{1}, t_{2}, \cdots, t_{n}\right] \in \mathrm{R}^{n}$. The latter, if $p \geqq 2$, follows immediately from (1.4) with $j=2$, which restricts $t$ to the sphere with radius $\left(n m_{2} / 2\right)^{1 / 2}$ and center at the origin. If $p<2$, we may argue that the objective function in (1.3) is never less than $\left[R_{n}\left(t^{2}\right)\right]^{2}=\left((2 / n) \sum_{k=1}^{n} t_{k}{ }^{2}-m_{2}\right)^{2}$ and thus tends with $\|t\|$ to infinity. It suffices, therefore, to consider Problem I on a sufficiently large, but finite, ball in $\mathrm{R}^{n}$. The existence of a solution then follows from the fact that we are minimizing a continuous function on a nonempty compact set. 
From the theory of Lagrange multipliers [1, p. 153] it follows that the minimum point either satisfies the equations

$$
\begin{gathered}
\frac{\partial}{\partial t_{i}}\left\{\sum_{i=p+1}^{n}\left[R_{n}\left(t^{j}\right)\right]^{2}-\sum_{j=1}^{p} \lambda_{j} R_{n}\left(t^{j}\right)\right\}=0, \quad i=1,2, \cdots, n, \\
R_{n}\left(t^{j}\right)=0, \quad j=1,2, \cdots, p,
\end{gathered}
$$

or else in such that the Jacobian matrix of the system (2.3) has rank $<p$. (In the case $p=0$, only (2.2), without multipliers, need be considered.) We first consider (2.2), (2.3). Carrying out the differentiations, we can write these equations more explicitly in the form

$$
\begin{gathered}
\sum_{i=p+1}^{n}\left(\frac{2}{n} \sum_{k=1}^{n} t_{k}^{i}-m_{i}\right) j t_{i}^{j-1}+\frac{1}{2} \sum_{j=1}^{p} \lambda_{j} j t_{i}^{i-1}=0, \quad i=1,2, \cdots, n, \\
\frac{2}{n} \sum_{k=1}^{n} t_{k}^{i}-m_{i}=0, \quad j=1,2, \cdots, p .
\end{gathered}
$$

We interpret (2.2') as a system of $n$ linear homogeneous equations in the $n$ "unknowns" $u_{i}=\frac{1}{2} j \lambda_{j} \quad(j=1,2, \cdots, p), \quad u_{i}=j\left(\frac{2}{n} \sum_{k=1}^{n} t_{k}^{j}-m_{j}\right) \quad(j=p+1, \cdots, n)$.

Not all of these can vanish, since otherwise $\left(2.3^{\prime}\right)$ would hold not only for $i=1,2, \cdots, p$, but also for $j=p+1, \cdots, n$, which is impossible by Bernstein's result. Consequently, the determinant of the system $\left(2.2^{\prime}\right)$, a Vandermondian, must vanish, giving

$$
\left|\begin{array}{ccccc}
1 & t_{1} & t_{1}^{2} & \cdots & t_{1}^{n-1} \\
1 & t_{2} & t_{2}^{2} & \cdots & t_{2}^{n-1} \\
\cdots & \cdots & \cdots & \cdots & \cdots \\
1 & t_{n} & t_{n}^{2} & \cdots & t_{n}^{n-1}
\end{array}\right|=\prod_{i>i}\left(t_{i}-t_{i}\right)=0 .
$$

There exists, therefore, at least one pair of distinct indices $i \neq j$ such that $t_{i}=t_{i}$, i.e., $t_{i}$ is a multiple node. The same conclusion is reached by considering the other alternative, concerning the rank of the Jacobian of $\left(2.3^{\prime}\right)$, since the latter is also a Vandermonde matrix. This completes the proof of Theorem 2.2.

It is likely that multiple nodes occur also for $q>n$, but we have no proof for this.

3. The Symmetric Problem. In view of (1.5) there are now only [ $n / 2]$ independent variables, which we assume ordered decreasingly,

$$
t_{1} \geqq t_{2} \geqq \cdots \geqq t_{\nu} \geqq 0, \quad \nu=[n / 2] .
$$

If $n$ is odd, one node, say $t_{v+1}$, must vanish and therefore does not appear among the independent variables (3.1).

We consider Problem $\mathrm{I}^{\prime}$ for $q=n$ only. Letting $\pi=[p / 2]$, the problem is then equivalent to

$$
\sum_{i=\pi+1}^{\infty}\left[R_{n}\left(t^{2 i}\right)\right]^{2}=\min , \quad R_{n}\left(t^{2 i}\right)=0, \quad j=1,2, \cdots, \pi
$$


since $R_{n}\left(t^{i}\right)=0$ for $j$ odd. We assume $\pi<\nu$ to insure a nonvoid objective function in (3.2). (If $n$ is even, this is always satisfied.) More explicitly, (3.2) can be written in the form

(3.2') $\sum_{i=\pi+1}^{\nu}\left(\frac{4}{n} \sum_{k=1}^{\nu} t_{k}^{2 j}-m_{2 i}\right)^{2}=\min , \frac{4}{n} \sum_{k=1}^{\nu} t_{k}^{2 i}-m_{2 i}=0, j=1,2, \cdots, \pi$.

THEOREM 3.1. Let $q=n$, and $n=8$ or $n \geqq 10$. If the constraints in (3.2') admit real solutions, then every solution of Problem $\mathrm{I}^{\prime}$ has at least one multiple node.

Proof. Existence and boundedness of a solution of Problem $I^{\prime}$ follows as in the proof of Theorem 2.2. If $t_{\nu}=0$, then the origin is a multiple node, since nodes occur in pairs, if $n$ is even, and include the extra node $t_{v+1}=0$, if $n$ is odd. Any solution with $t_{v}>0$, on the other hand, by the theory of Lagrange multipliers must satisfy

$$
\sum_{i=x+1}^{\infty}\left(\frac{4}{n} \sum_{k=1}^{\nu} t_{k}^{2 i}-m_{2 i}\right) j t_{i}^{2 i-1}+\frac{1}{2} \sum_{i=1}^{\pi} \lambda_{i} j t_{i}^{2 i-1}=0, \quad i=1,2, \cdots, \nu,
$$

or be such that the Jacobian matrix of the constraint equations has rank $<\pi$. As before, this implies

$$
\left|\begin{array}{cccc}
t_{1} & t_{1}^{3} & \cdots & t_{1}^{2 \nu-1} \\
t_{2} & t_{2}^{3} & \cdots & t_{2}^{2 \nu-1} \\
\cdots & \cdots & \cdots & \cdots \\
t_{\nu} & t_{\nu}^{3} & \cdots & t_{\nu}^{2 \nu-1}
\end{array}\right|=t_{1} t_{2} \cdots t_{\nu} \prod_{i>i}\left(t_{i}^{2}-t_{i}^{2}\right)=0 .
$$

Since $t_{v}>0$, we conclude $t_{i}{ }^{2}=t_{i}{ }^{2}$ for some $i \neq j$, and thus $t_{i}=t_{i}$, by virtue of (3.1). This proves Theorem 3.1.

In the case $\pi=\nu-1$ (considered by Kahaner [4]), the method of Lagrange multipliers is actually more powerful. It permits us to reduce the problem to the much simpler problem of solving at most $\pi+2$ single algebraic equations of degree $\leqq \pi$. The following theorem is a step in this direction.

THEOREM 3.2. Let $q=n, n=8$ or $n \geqq 10$, and $\pi=\nu-1$, where $\pi=[p / 2]$, $\nu=[n / 2]$. If Problem $\mathrm{I}^{\prime}$ admits solution, then one or both of the following conditions must hold:

(A) The system of equations

$$
\sum_{k=1}^{\pi} x_{k}^{j}=\frac{n}{4} m_{2 i}, \quad j=1,2, \cdots, \pi,
$$

has nonnegative solutions.

(B) The system of equations

$$
\sum_{k=1}^{\pi-1} x_{k}^{i}+2 x_{\pi}^{j}=\frac{n}{4} m_{2 j}, \quad j=1,2, \cdots, \pi,
$$

has nonnegative solutions.

Putting

$$
t_{k}^{*}=x_{k}^{1 / 2} \quad(k=1,2, \cdots, \pi), \quad t_{\nu}^{*}=0
$$

for a solution in case (A), and

$$
t_{k}^{*}=x_{k}^{1 / 2} \quad(k=1,2, \cdots, \pi-1), \quad t_{\pi}^{*}=t_{\nu}^{*}=x_{\pi}^{1 / 2}
$$


for a solution in case (B), let $\left\{t_{k}\right\}_{k=1}^{\nu}$ denote the permutation of $\left\{t_{k}^{*}\right\}_{k=1}^{\nu}$ which satisfies (3.1). The solution of problem (3.2') is then given by that set of nodes $t_{1}, t_{2}, \cdots, t_{v}$ which gives the smallest value to the objective function in $\left(3.2^{\prime}\right)$. If the nodes derive from case (A), then the solution to Problem $\mathrm{I}^{\prime}$ has a multiple node at the origin; if they derive from case (B), then there is a nonzero multiple node among the solution.

Proof. We already know from the proof of Theorem 3.1 that the solution $\mathrm{t}=\left[t_{1}, t_{2}, \cdots, t_{\nu}\right]$ of problem $\left(3.2^{\prime}\right)$ must satisfy (3.3) and the constraints in $\left(3.2^{\prime}\right)$. Corresponding to the $\nu(\nu+1) / 2$ factors in (3.3), there are as many possibilities to consider, which in view of (3.1) we may list as follows in terms of $x_{k}=t_{k}{ }^{2}$ :

$$
\begin{aligned}
& x_{v}=0 \quad(1,1) \\
& x_{\nu-1}=x_{\nu}=0 \quad(1,2) \\
& \ldots \ldots \ldots \ldots \\
& x_{1}=x_{2}=\cdots=x_{\nu}=0 \quad(1, \nu) \\
& x_{\nu-1}=x_{\nu} \quad(2,1) \\
& x_{\nu-2}=x_{\nu-1}=x_{\nu} \quad(2,2) \\
& \ldots \ldots \ldots \ldots \ldots \\
& x_{1}=x_{2}=\cdots=x_{\nu-1}=x_{\nu} \quad(2, \nu-1) \\
& x_{\nu-2}=x_{\nu-1} \quad(3,1) \\
& x_{\nu-3}=x_{\nu-2}=x_{\nu-1} \quad(3,2) \\
& \text {................... } \\
& x_{1}=x_{2}=\cdots=x_{\nu-2}=x_{\nu-1} \quad(3, \nu-2) \\
& x_{1}=x_{2} \quad(\nu, 1)
\end{aligned}
$$

Condition $(1,1)$, together with the constraints in $\left(3.2^{\prime}\right)$, gives the system in case (A) of the theorem. The subsequent conditions $(1,2), \cdots,(1, \nu)$ simply imply that this system has a solution with one or more zero components. (We see later in examples that this is not possible.) Condition $(2,1)$, together with the constraints, gives rise to the system of case (B). The subsequent conditions through $(2, \nu-1)$ simply mean that this system has multiple solutions. The conditions in group 3 are either a permutation of those in group 2, or in fact identical with them if $x_{\nu-1}=x_{\nu}$. Similar arguments apply to all subsequent groups. We thus see that if $\mathrm{t}=\left[t_{1}, t_{2}, \cdots, t_{v}\right]$ is a solution of (3.1), $\left(3.2^{\prime}\right)$, then the corresponding $\mathrm{x}=\left[x_{1}, x_{2}, \cdots, x_{\nu}\right]$ (with $x_{k}=t_{k}{ }^{2}$ ) is such that either $x_{v}=0$ and the remaining components solve the system of case (A), or there is a permuted vector $\mathrm{x}$, say, $\mathrm{x}^{*}=\left[x_{1}{ }^{*}, x_{2}{ }^{*}, \cdots, x_{\nu-1}{ }^{*}, x_{\nu}{ }^{*}\right]$ such that $x_{\nu-1}{ }^{*}=x_{\nu}{ }^{*}$ and $x_{1}{ }^{*}, x_{2}{ }^{*}, \cdots, x_{\pi}{ }^{*}$ solves the system of case (B). This proves the part of Theorem 3.2 concerning (A) and (B). The rest of the theorem is a consequence of Lagrange multiplier theory.

The solution of the systems in (A) and (B) of Theorem 3.2 is discussed in the next section. 
4. The Solution of Some Auxiliary Systems of Algebraic Equations. We consider first the system

$$
\sum_{k=1}^{n} x_{k}^{i}=s_{i}, \quad j=1,2, \cdots, n
$$

The lemma which follows is well known.

LEMMA 4.1. (NEWTON'S IDENTITIES). If $\left\{x_{k}\right\}_{k=1}^{n}$ is a solution of (4.1) and

$$
\xi(x)=\prod_{k=1}^{n}\left(x-x_{k}\right)=x^{n}+a_{1} x^{n-1}+\cdots+a_{n},
$$

then

$$
\begin{aligned}
& s_{1}+a_{1}=0 \\
& s_{2}+a_{1} s_{1}+2 a_{2}=0, \\
& \ldots \ldots \ldots \ldots \cdots \cdots+a_{n-1} s_{1}+n a_{n}=0 . \\
& s_{n}+a_{1} s_{n-1}+\cdots+
\end{aligned}
$$

Conversely, if the constants $\left\{a_{k}\right\}_{k=1}^{n}$ satisfy (4.3), then the zeros of $\xi(x)$ in (4.2) solve (4.1).

The solution of $(4.1)$ is thus reduced to the solution of a single algebraic equation, viz., $\xi(x)=0$.

Together with (4.1) we now consider the system

$$
\sum_{k=1}^{n} y_{k}^{i}=s_{i}-z^{i}, \quad j=1,2, \cdots, n,
$$

where $z$ is an arbitrary parameter.

LEMMA 4.2. Let $\left\{x_{k}\right\}_{k=1}^{n},\left\{y_{k}\right\}_{k=1}^{n}$ be solutions of (4.1) and (4.4), respectively. If

$$
\begin{aligned}
& \xi(x)=\prod_{k=1}^{n}\left(x-x_{k}\right)=x^{n}+a_{1} x^{n-1}+\cdots+a_{n}, \\
& \eta(x)=\prod_{k=1}^{n}\left(x-y_{k}\right)=x^{n}+b_{1} x^{n-1}+\cdots+b_{n},
\end{aligned}
$$

then

$$
b_{r}=z^{r}+a_{1} z^{r-1}+\cdots+a_{r}, \quad r=1,2, \cdots, n .
$$

Proof. By virtue of (4.1) and (4.4), we have, for large $x$,

$$
\begin{aligned}
& \frac{\xi^{\prime}(x)}{\xi(x)}=\sum_{k=1}^{n} \frac{1}{x-x_{k}}=\sum_{j=0}^{n} \frac{s_{j}}{x^{j+1}}+O\left(x^{-n-2}\right), \\
& \frac{\eta^{\prime}(x)}{\eta(x)}=\sum_{k=1}^{n} \frac{1}{x-y_{k}}=\sum_{j=0}^{n} \frac{s_{j}}{x^{j+1}}-\sum_{j=1}^{n} \frac{z^{j}}{x^{j+1}}+O\left(x^{-n-2}\right),
\end{aligned}
$$

where $s_{0}=n$. Consequently,

$$
\frac{\eta^{\prime}(x)}{\eta(x)}=\frac{\xi^{\prime}(x)}{\xi(x)}-\left(\frac{1}{x-z}-\frac{1}{x}\right)+O\left(x^{-n-2}\right),
$$

which, upon integration, yields 


$$
\eta(x)=\text { const } \frac{x \xi(x)}{x-z}\left[1+O\left(x^{-n-1}\right)\right] .
$$

Since $\eta(x)$ and $\xi(x)$ are both polynomials with leading coefficient 1 , the constant in this identity is 1 , and we get

$$
(x-z) \eta(x)=x \xi(x)\left[1+O\left(x^{-n-1}\right)\right] .
$$

Comparing coefficients of the powers $x^{n}, x^{n-1}, \cdots, x$ on either side, we find

$$
a_{r}=b_{r}-z b_{r-1}, \quad r=1,2, \cdots, n,
$$

where $b_{0}=1$, which, when solved for the $b$ 's, gives (4.7).

LEMMA 4.3. Let $\left\{x_{k}\right\}_{k=1}^{n},\left\{y_{k}\right\}_{k=1}^{n}$ be solutions, respectively, of

$$
\sum_{k=1}^{n} x_{k}^{j}=s_{i}, \quad \sum_{k=1}^{n-1} y_{k}^{j}+2 y_{n}^{i}=s_{i}, \quad j=1,2, \cdots, n,
$$

and

$$
\xi(x)=\prod_{k=1}^{n}\left(x-x_{k}\right)=x^{n}+a_{1} x^{n-1}+\cdots+a_{n} .
$$

Then $y_{n}$ is a root of the algebraic equation

$$
(d / d y)[y \xi(y)]=0 .
$$

Proof. Put $z=y_{n}$ in Lemma 4.2. The system (4.4) then becomes the second system in (4.8), and from (4.6) we see that $\eta\left(y_{n}\right)=0$, i.e., in view of (4.7),

$$
\sum_{r=0}^{n}\left(\sum_{s=0}^{r} a_{s} y_{n}^{r-s}\right) y_{n}^{n-r}=0
$$

Inverting the order of summation, we get

$$
\sum_{s=0}^{n}(n-s+1) a_{s} y_{n}^{n-s}=0,
$$

which is the desired result.

Lemma 4.3 suggests a method for solving the second system in (4.8). We first solve (4.10), where Lemma 4.1 is used to obtain $\xi(x)$. For each root $y_{n}$ of $(4.10)$, we then solve

$$
\sum_{k=1}^{n-1} y_{k}^{i}=s_{j}-2 y_{n}^{i}, \quad j=1,2, \cdots, n-1,
$$

which is again a problem of the type considered in Lemma 4.1.

We note that if the equation $\xi(x)=0$ has $m(\leqq n)$ distinct real nonvanishing roots, then Eq. (4.10) has at least $m$ such roots. This is a simple consequence of Rolle's theorem.

5. Numerical Results. According to Bernstein [cf. (1.2)] Problem $\mathrm{I}^{\prime}$ for $q=n$, $\pi=\nu-1$ cannot have a real solution if $n-2 \geqq 4 n^{1 / 2}$, i.e., if $n \geqq 20$. Hence, we explore the integers $n=8$ and $10 \leqq n \leqq 19$ for possible real solutions, following the procedures outlined in Theorem 3.2 and Lemmas 4.1, 4.3. It turns out that the problem 
TABLE 1.

Search for optimal nodes

\begin{tabular}{|c|c|c|c|c|c|c|}
\hline$n$ & $\pi$ & primary $\xi$ & object. fct. & $(x \xi)^{\prime}$ & secondary $\xi$ & object. fct. \\
\hline & & & (in $10^{-7}$ ) & & & (in $10^{-7}$ ) \\
\hline \multirow[t]{3}{*}{8} & 3 & .809 & & .659 & no pos. zeros & - \\
\hline & & .328 & & .266 & 1 pos. zero & - \\
\hline & & .197 & 40.9 & .074 & $.801, .384$ & 293. \\
\hline \multirow[t]{4}{*}{10} & 4 & .846 & & .730 & no pos. zeros & - \\
\hline & & .439 & & .375 & 1 pos. zero & - \\
\hline & & .288 & & .190 & 2 pos. zeros & - \\
\hline & & .093 & 23.5 & .038 & $.847, .416, .326$ & 14.2 \\
\hline \multirow[t]{4}{*}{11} & 4 & 2 pos. zeros & - & .750 & no pos. zeros & - \\
\hline & & & & .419 & 1 pos. zero & - \\
\hline & & & & .228 & $.856, .514, .008$ & 24.1 \\
\hline & & & & .070 & $.860, .455, .378$ & 3.27 \\
\hline \multirow[t]{5}{*}{12} & 5 & 1 pos. zero & - & .777 & no pos. zeros. & - \\
\hline & & & & .456 & 2 pos. zeros & - \\
\hline & & & & .297 & 2 pos. zeros & - \\
\hline & & & & .097 & 2 pos. zeros & - \\
\hline & & & & .040 & 2 pos. zeros & - \\
\hline \multirow[t]{5}{*}{13} & 5 & 1 pos. zero & - & .791 & no pos. zeros & - \\
\hline & & & & .491 & $\begin{array}{l}.882, .159, .118 \\
.026\end{array}$ & 1.48 \\
\hline & & & & .327 & 2 pos. zeros & - \\
\hline & & & & .139 & 2 pos. zeros & - \\
\hline & & & & .057 & $\begin{array}{l}.882, .525, .450 \\
.197\end{array}$ & 1.94 \\
\hline \multirow[t]{4}{*}{14} & 6 & 2 pos. zeros & - & .811 & no pos. zeros & - \\
\hline & & & & .516 & 2 pos. zeros & - \\
\hline & & & & .390 & 2 pos. zeros & - \\
\hline & & & & .011 & 1 pos. zero & - \\
\hline \multirow[t]{6}{*}{15} & 6 & 2 pos. zeros & - & .822 & no pos. zeros & - \\
\hline & & & & .545 & 2 pos. zeros & - \\
\hline & & & & .413 & 2 pos. zeros & - \\
\hline & & & & .183 & 2 pos. zeros & 一 \\
\hline & & & & .148 & 2 pos. zeros & - \\
\hline & & & & .032 & 1 pos. zero & - \\
\hline \multirow[t]{3}{*}{16} & 7 & 1 pos. zero & - & .836 & no pos. zeros & - \\
\hline & & & & .560 & 2 pos. zeros & - \\
\hline & & & & .470 & 2 pos. zeros & - \\
\hline \multirow[t]{3}{*}{17} & 7 & 1 pos. zero & - & .845 & no pos. zeros & - \\
\hline & & & & .586 & 2 pos. zeros & - \\
\hline & & & & .486 & 2 pos. zeros & - \\
\hline \multirow[t]{3}{*}{18} & 8 & 1 pos. zero & - & .856 & no pos. zeros & - \\
\hline & & & & .592 & 2 pos. zeros & - \\
\hline & & & & .541 & 2 pos. zeros & - \\
\hline \multirow[t]{4}{*}{19} & 8 & 2 pos. zeros & - & .862 & no pos. zeros & - \\
\hline & & & & .617 & 2 pos. zeros & - \\
\hline & & & & .550 & 2 pos. zeros & - \\
\hline & & & & .009 & 1 pos. zero & - \\
\hline
\end{tabular}

again has no solution if $n=12$ or $n \geqq 14$. The results of our computation are summarized in Table 1. In the first two columns of this table we record the values of $n$ 
and $\pi=[n / 2]-1$, respectively. The third column provides information concerning the positive zeros* of the "primary" polynomial $\xi$. This is the polynomial $\xi$ (of degree $\pi$ ) defined in Lemma 4.1, which corresponds to the system (A) of Theorem 3.2. We list all its positive zeros, if there are $\pi$ of them, and only state their number, otherwise. A complete set of $\pi$ positive zeros of $\xi$ constitutes a candidate for an optimal solution. The fourth column contains the corresponding value of the objective function in $\left(3.2^{\prime}\right)$. In the next column are listed all positive zeros* of $(x \xi)^{\prime}$ [cf. Lemma 4.3]. To each of these zeros corresponds a "secondary" polynomial $\xi$ (of degree $\pi-1$ ), which is the $\xi$-polynomial belonging to the system (4.11). Information concerning its positive zeros* is given in column six. Any complete set of $\pi-1$ positive zeros, together with the corresponding zero of $(x \xi)^{\prime}$, again constitutes a candidate for an optimal solution. The corresponding value of the objective function is given in the last column. Entries printed in italic type correspond to optimal solutions.

We note that all zeros listed in Table 1 correspond to $x_{k}$-values in Theorem 3.2; the desired nodes are the square roots $t_{k}=x_{k}{ }^{1 / 2}$. The optimal nodes to 25 decimals, as computed in double precision arithmetic on the CDC 6500 computer, are as follows:

$$
\begin{aligned}
& n=8 \\
& \begin{array}{llllll}
-t_{8}=t_{1}=.89917 & 93430 & 79596 & 08730 & 30645
\end{array} \\
& \begin{array}{llllll}
-t_{7}=t_{2}=.57261 & 84272 & 82722 & 13153 & 89072
\end{array} \\
& \begin{array}{llllll}
-t_{6}=t_{3}=.44375 & 44129 & 90497 & 74722 & 36463
\end{array} \\
& -t_{5}=t_{4}=0 \\
& \begin{array}{lllll}
n=10 \\
-t_{10}=t_{1}=.92019 & 94551 & 46763 & 72230 & 02697
\end{array} \\
& \begin{array}{llllll}
-t_{9}=t_{2}=.64533 & 80565 & 58410 & 15617 & 64507
\end{array} \\
& \begin{array}{llllll}
-t_{8}=t_{3}=.57137 & 69273 & 35333 & 94337 & 27524
\end{array} \\
& -t_{7}=t_{4}=.19617 \quad 1900485916 \quad 40260 \quad 18800=-t_{6}=t_{5} \\
& -t_{11}=t_{1}=.92750 \quad 15617 \quad 64337 \quad 25089 \quad 95943 \\
& -t_{10}=t_{2}=.67480 \quad 04201 \quad 44051 \quad 83360 \quad 39625 \\
& -t_{9}=t_{3}=.61476 \quad 53471 \quad 66840 \quad 31224 \quad 97967 \\
& -t_{8}=t_{4}=.2643691993 \quad 73049 \quad 18149 \quad 65951=-t_{7}=t_{5} \\
& t_{6}=0 \\
& n=13 \\
& \begin{array}{llllll}
-t_{13}=t_{1}=.93913 & 47142 & 41241 & 62154 & 14039
\end{array} \\
& -t_{12}=t_{2}=.70075 \quad 43220 \quad 36606 \quad 99992 \quad 84358=-t_{11}=t_{3} \\
& -t_{10}=t_{4}=.39893 \quad 86129 \quad 44348 \quad 55731 \quad 47686 \\
& -t_{9}=t_{5}=.34310 \quad 66664 \quad 50818 \quad 91837 \quad 46133 \\
& -t_{8}=t_{6}=. \begin{array}{lllll}
16032 & 84566 & 34386 & 73493 & 97197
\end{array} \\
& t_{7}=0
\end{aligned}
$$

Comparison with Table 2 in [5] reveals agreement in all twelve digits given there.

* None of the zeros vanish; we could thus equally well speak of "nonnegative" zeros. 
Department of Computer Sciences

Purdue University

Lafayette, Indiana 47907

Department of Mathematics

Fukuoka University of Education

Munakata, Fukuoka, 811-41

Japan

1. T. M. Apostol, Mathematical Analysis: A Modern Approach to Advanced Calculus, Addison-Wesley, Reading, Mass., 1957. MR 19, 398.

2. R. E. Barnhill, J. E. Dennis, JR. \& G. M. Nielson, "A new type of Chebyshev quadrature," Math. Comp., v. 23, 1969, pp. 437-441. MR 39 \#3698.

3. S. N. BERNŠTEǏn, "Sur les formules de quadrature de Cotes et Tchebycheff," $C$. $R$. Acad. Sci. URSS, v. 14, 1937, pp. 323-326; Reprinted in Collected Works. Vol. II, Izdat. Akad. Nauk SSSR, Moscow, 1954, pp. 200-204. (Russian) MR 16, 433.

4. D. Kahaner, "Chebyshev type quadrature formulas," Math. Comp., v. 24, 1970, pp. 571-574. MR 42 \#8694.

5. P. Rabinowitz \& N. Richter, "Chebyshev-type integration rules of minimum norm," Math. Comp., v. 24, 1970, pp. 831-845. MR 45 \#7996. 